\title{
Una experiencia de teleformación del profesorado de Educación Física (Primaria y Secundaria) en actividades físicas en el medio natural Formation on line an experience of the faculty of Physical Education
(Primary and Secondary) in Physical Activity in the Natural Environment
}

\author{
Antonio Granero Gallegos \\ Universidad de Almería (España)
}

\begin{abstract}
Resumen: Las nuevas tecnologías de la información y la comunicación son una realidad que hay que afrontar, también desde la formación del profesorado de primaria y secundaria, pues representan un recurso cada día más imprescindible en nuestra vida y unas herramientas adecuadas para la preparación presente y futura del alumnado. Desde el Centro del Profesorado Cuevas-Olula (Almería) se ha realizado una actividad de teleformación dirigida al profesorado de Educación Física, trabajando distintos contenidos curriculares de actividades en el medio natural, y buscando poner de manifiesto que todas las áreas del conocimiento pueden y deben adaptarse a las nuevas tecnologías. Esta actividad de formación demuestra el gran interés e implicación de maestros y profesores de Educación Física en la propia actualización y utilización de los recursos TIC. La evaluación del curso, realizada por los propios participantes, evidencia la validez y el éxito del modelo de formación utilizado, con una parte teórico-práctica desarrollada on-line y con actividades prácticas presenciales al final, así como la valoración de las virtudes de la formación a distancia en comparación con la presencial y tradicional.
\end{abstract}

Palabras clave: formación del profesorado, TIC, Educación Física, actividades en el medio natural.

Abstract: The new technologies of information and communication are a reality that must be confronted, from teacher training to primary and secondary schools, since they represent a resource increasingly indispensable in our lives and about proper tools for the preparation of the present and future students. From the Center Teacher Cuevas-Olula (Almeria) has been engaged in an activity distance to the faculty of Physical Education, working different curricula activities in the natural environment, and seeking to demonstrate that all areas of knowledge can and should adapt to new technologies. This training demonstrates the keen interest and involvement of teachers and professors of Physical Education at the updating and resource use ICT. The course evaluation, conducted by the participants themselves, will evidence the validity and success of the training model used, with a party line developed theoretical and practical sessions at the end, as well as the valuation of the virtues of distance learning compared with the personal and traditional one.

Key words: Teacher training, ICT, Physical Education, activities in the natural environment.

\section{Introducción}

En los últimos años se está produciendo una gran potenciación en el uso de las denominadas nuevas tecnologías de la información y la comunicación (TIC) por parte de la administración pública. Son destacables, especialmente, las distintas convocatorias anuales que se realizan por parte de la Consejería de Educación de la Junta de Andalucía y que manan del Decreto 72/2003, de 18 de marzo, de Medidas de Impulso de la Sociedad del Conocimiento, en el seno del Sistema Andaluz de Formación del Profesorado, regulado por el Decreto 110/2003, de 22 de abril, y con motivo del desarrollo del II Plan Andaluz de Formación del Profesorado (Orden de 9 de junio de 2003), teniendo por objeto llegar a implicar en el uso de las TIC a la mayor parte del profesorado no universitario.

Como indican Palomo, Ruiz y Sánchez (2005), las citadas convocatorias cristalizan en compromisos por parte de los centros docentes, marcados por el deseo de mejora y guiados por el convencimiento de que las TIC representan un recurso cada día más imprescindible en nuestra vida y unas herramientas adecuadas para la preparación presente y futura del alumnado. Con todo, estas iniciativas suponen un esfuerzo por parte del profesorado, sobre todo en su propia formación, pues en muchos casos han de enfrentarse a cambios metodológicos, a nuevas aplicaciones informáticas, nuevos retos, cambio de mentalidad y de perspectiva respecto a los contenidos a impartir, a romper estructuras y esquemas, etc. Para ello, aseguran Cabero, Duarte y Barroso (1997), es necesaria una actitud convencida y favorable por parte del profesorado para la incorporación de estas tecnologías en la práctica docente.

Fecha recepción: 04-12-07 - Fecha envío revisores: 04-12-07 - Fecha de aceptación: 22-12-07 Correspondencia: Antonio Granero Gallegos

C/Mayor, $\mathrm{n}^{\circ} 1-$ bis, $4^{\circ} \mathrm{B}$

04630, Garrucha - Almería (España)

E-mail: agranero@ual.es
Con estos recursos se abren nuevas, interesantes y sugerentes posibilidades en el proceso de enseñanza-aprendizaje que, sin duda, ofrecen una abundante serie de ventajas para el alumnado, pero también para el profesorado (Majó y Marqués, 2002) pues, entre innumerables aspectos, representan una fuente de recursos educativos para el ejercicio de la docencia que suponen un gran paso adelante respecto a los tradicionales, la formación unidireccional deja paso a modelos más abiertos y flexibles, permiten la individualización de la enseñanza, a la vez que facilita la diversidad de agrupamientos para el desempeño de diferentes tareas, liberan de trabajos repetitivos, monótonos y rutinarios, facilitan la evaluación y control, así como la actualización profesional y conocimiento de múltiples experiencias didácticas, etc.

Es interesante poner un gran énfasis en la importancia que adquieren estas nuevas tecnologías en la formación del profesorado, no sólo en la inicial, sino que resulta fundamental en la formación permanente, pues, como indican algunos autores como Marcelo y Estebaranz (1999), los conocimientos adquiridos por el profesorado en la formación inicial se quedan obsoletos rápidamente si el docente deja de preocuparse por seguir aprendiendo.

En cualquier caso, no se debe olvidar que estos medios y recursos han de estar al servicio del profesorado, utilizándose en función de las necesidades, del nivel, etc. El uso de estas herramientas debe obedecer a unos criterios lógicos, cuidando, asimismo, que su utilización por parte del alumnado sea racional y efectiva, intentando encauzar y educar el uso de la amplia oferta al alcance de los adolescentes, pues en algunas investigaciones, como la de Foster, Campbelly Twenge (2003)y Twenge et al. (2007), se pone de manifiesto el notorio aumento del egocentrismo y narcisismo entre los jóvenes actuales, y este ego exacerbado conllevará, afirman, a un deterioro de las relaciones personales y sociales, multiplicando los problemas. Los centros -el profesorado, por ende-, como expone Cebrián de la Serna (1997), han de convertirse en espacios creadores de conocimiento, en verdaderos capacitadores de unos ciudadanos y profesionales críticos en el consumo de tecnología. 
Con todo, no cabe duda de que con la llegada y plena incorporación de las TIC a los centros, y a la vida de los docentes, -y discentes-, aparecen nuevas necesidades de formación, prácticamente día a día, con lo que la formación continua y permanente por parte del profesorado se hace imprescindible, para adaptarse de forma acertada a los nuevos y constantes cambios que experimenta la sociedad y desempeñar adecuadamente su función docente. De esta manera, como exponen Palomo et al. (2005), para que el Plan de Formación del Profesorado se desarrolle de forma acertada y dé respuestas a las nuevas necesidades, se debe incentivar la formación desde los distintos Centros del Profesorado. En este sentido, se hace especial hincapié en la denominada formación en Centros, pero es indudable, dado lo expuesto con anterioridad, la necesidad de ofrecer al profesorado la posibilidad de seguir ampliando y actualizando su formación inicial.

Se debe incentivar, así, la formación del profesorado en el uso didáctico de estas nuevas tecnologías, pero no cometamos el error de pensar que el elemento más valioso en el proceso de enseñanza-aprendizaje son los recursos tecnológicos, sino que el profesorado sigue siendo la piedra angular del proceso, adaptado, obviamente, a las nuevas circunstancias, adoptando nuevos roles y funciones. Lo expresa muy bien Cebrián de la Serna (1997,p. 1), al afirmar que «hoy, la calidad del producto educativo radica más en la formación permanente e inicial del profesorado que en la sola adquisición y actualización de infraestructuras».

En el presente artículo se pretende dar a conocer una experiencia de teleformación (formación on-line) desarrollada con el profesorado de Educación Física, tanto de primaria como de secundaria. Y es que, aunque a veces puede parecer que desarrolla su labor docente ajeno a estas nuevas tecnologías, también forma parte de la denominada Sociedad del Conocimiento y ha de realizar esfuerzos por formarse y actualizar conocimientos para el desempeño de su labor en el aula. Así, en el Plan de Formación del Centro del Profesorado Cuevas-Olula (Almería) se decide incluir una actividad formativa dirigida a este colectivo(Educación Física) con la peculiaridad de que se desempeño como formación on line o teleformación, a través de la Plataforma Educativa Moodle. De esta manera, también desde el CEP se intenta poner de manifiesto que todas las áreas de conocimiento han de adaptarse a las nuevas tecnologías y, en este caso, aunque los contenidos del área sean eminentemente prácticos, tienen una importante base teórica sobre la que trabajar y sobre la que desarrollar diferentes propuestas de praxis.
Asimismo, el curso planteado constituía el reto de ser pionero en trabajar diversos contenidos de actividades en el medio natural a través de la teleformación y con profesorado de toda la provincia. Uno de los objetivos que se planteó el CEP al decidir llevar a cabo esta actividad formativa fue poner en contacto, buscando la interacción y el intercambio de experiencias, vía Internet, al profesorado de Educación Física de la provincia deAlmería mediante la citada plataforma educativa.

\section{Planeamiento de la actividad}

La denominación con que se hizo pública la convocatoria de esta actividad de formación permanente del profesorado ( $«$ Las actividades en el medio natural como contenido del área de Educación Física») da claras pistas de los contenidos trabajados y de los destinatarios, especialistas de toda la provincia de Almería de éste área de conocimiento. Siempre teniendo en cuenta los recientemente publicados Reales Decretos que establecen las Enseñanzas Mínimas, tanto de Educación Primaria, R.D. 1513/2006, de 7 de diciembre, como de Educación Secundaria Obligatoria, R.D. 1631/2006, de 29 de diciembre, estableciendo el currículum de cada una de las áreas. Aunque en un principio se pensó en desarrollar el curso solamente dentro del ámbito de actuación del CEP Cuevas-Olula, finalmente, dadas las características de la actividad, una vez finalizado su diseño, se optó por abrirla y ofertarla a toda la provincia, incluyendo así la oferta al profesorado del CEP de Almería y El Ejido. De esta manera, el curso queda enclavado para la segunda convocatoria del Plan Provincial de Teleformación «CEPIndalo», desarrollado a través de la Plataforma Moodle.

\subsection{Objetivos}

Los objetivos pretendidos en el desarrollo de esta actividad formativa son los siguientes:

- Conceptuar las causas geográficas que determinan los conceptos de orientación espacial y temporal.

- Conocer las distintas representaciones de la tierra en cartas, planos y/o mapas topográficos.

- Orientarse en diferentes situaciones a partir de datos obtenidos con instrumentos, mapas y signos naturales.

- Organizar un campamento y elaborar el programa de actividades según las características del grupo y objetivos propuestos.

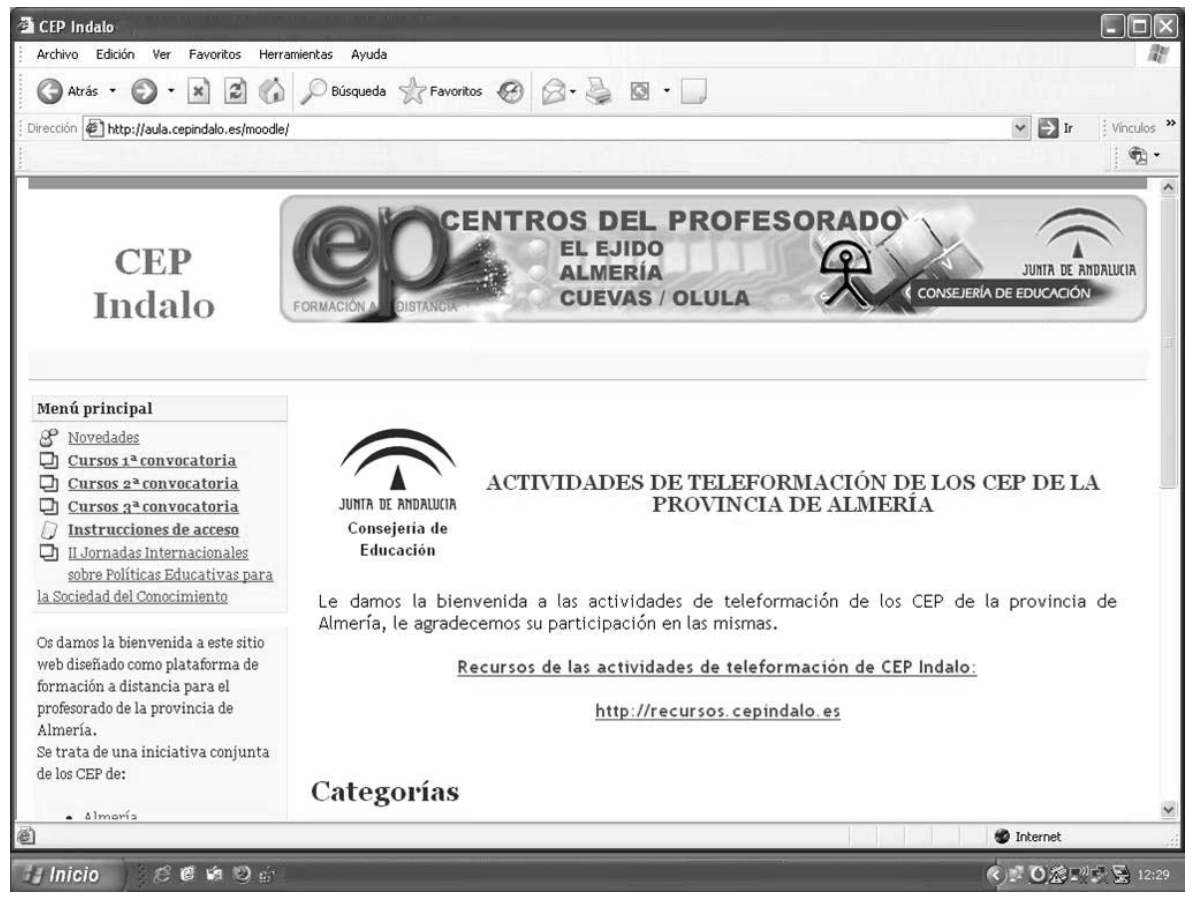

Figura 1 - Web de la Plataforma Provincial de Teleformación (CEPIndalo, 2007). 
- Conocery llevar a la práctica los aspectos básicos de las actividades relacionadas con la bicicleta (su mecánica y mantenimiento).

- Analizar las características del medio, las técnicas de progresión y desplazarse en diferentes tipos de terreno utilizando material adecuado.

\subsection{Contenidos}

De forma sintética se exponen los contenidos de trabajo, estructurados en tres bloques de trabajo:

* Bloque 1:

- Cartografia y orientación: técnicas de navegación, técnicas de orientación.

- Organización de campamentos y técnicas de acampada.

- Aplicaciones didácticas.

*Bloque 2:

- Actividades con bicicleta: material y equipamiento, técnicas de conducción, mecánica, mantenimiento y organización de itinerarios.

* Bloque 3

- Aplicaciones didácticas.

- Técnicas de marcha y travesía: marcha, senderismo, acampada y vivac.

- Distintas técnicas de actividades físico-deportivas en el medio natural.

-Aplicaciones didácticas.

\section{Desarrollo del curso}

La actividad se programó con una duración total de 40 horas para desarrollar los distintos contenidos expuestos. De las 129 solicitudes recibidas se seleccionaron, teniendo en cuenta los criterios expuestos en la convocatoria, a 90 participantes, de los cuáles, 42 imparten docencia en secundaria y 46 son maestros de primaria. Teniendo en cuenta la variables sexo, indicar que del total de participantes que inició el curso, 60 eran varones y 30 , mujeres.

\subsection{La parte on-line}

Tutorizada por tres profesores, se realizó desde el 9 de enero hasta el 27 de febrero de 2007 a través de la Plataforma Provincial de
Teleforamción: «CEPIndalo», que engloba las propuestas de actividades formativas a distancia dirigidas al profesorado de los tres CEP de la provincia de Almería. Durante esta fase se trabajaron los contenidos expuestos en los tres bloques citados, mediante actividades teóricoprácticas que el alumnado había de realizar, tras «bajarse» y trabajar los temas, y «subir» a la plataforma para que fueran corregidas por los tutores. Asimismo, se plantaron actividades de evaluación de cada contenido tratado.

Se habilitaron varios foros (general y por temáticas) para que el alumnado pudiese participar e interconectar entre sí y con los tutores, salas de Chat, espacios para bajarse y subir archivos que pudieran resultar de interés para el resto de compañeros, direcciones Web para completar y ampliar contenidos, etc.; de esta manera se podían plantear y resolver dudas, así como aquellas cuestiones que se consideraran oportunas.

\subsection{La parte presencial}

Se programó, para completar la parte teórico-práctica «on-line», una presencial que se desarrolló durante los días 14 y 15 de abril de 2007, en el Parque Natural de la Sierra de Baza [figura 3], utilizando como alojamiento, lugar de convivencia y centro de operaciones, un complejo de turismo rural, denominado Cortijo Narváez. Se realizaron actividades prácticas sobre los contenidos tratados en la parte teórica a distancia y sirvió como punto de encuentro, intercambio y convivencia entre el profesorado de Educación Física participante, aunque en esta ocasión ejercieran de alumnado. El alumnado se repartió en tres grupos, para ir rotando por las diferentes actividades.

Se prepararon tres prácticas diferentes, cada una relacionada con uno de los bloques de contenidos trabajados, pero todas girando en torno a la orientación. Para el desarrollo de las mismas se repartió al alumnado en tres grandes grupos, para ir rotando por las diferentes actividades que se realizaban de forma simultánea. A su vez, para cada actividad se subdividieron en grupos de cuatro personas, teniendo en cuenta que no se podían separar durante la realización de la práctica, por lo que se recomendaba el agrupamiento por niveles. Las recomendaciones iniciales respecto a organización y seguridad fueron las propias de este tipo de actividades.

En relación al primer bloque se preparó una práctica de cartografía y orientación, lo que se denomina «score orienteering», entregando a

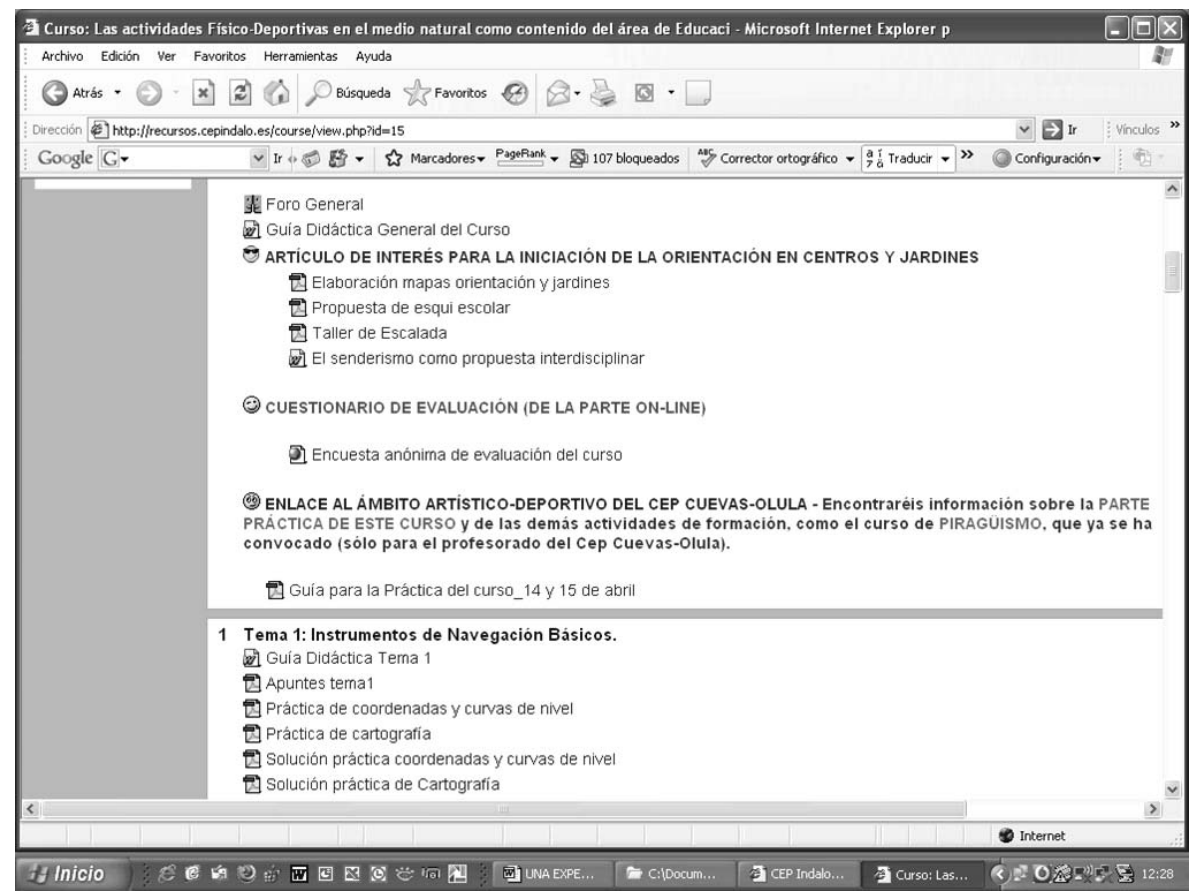

Figura 2 - Web del curso de formación Las actividades físicas en el medio natural como contenido del área de Educación Física (CEPIndalo, 2007). 


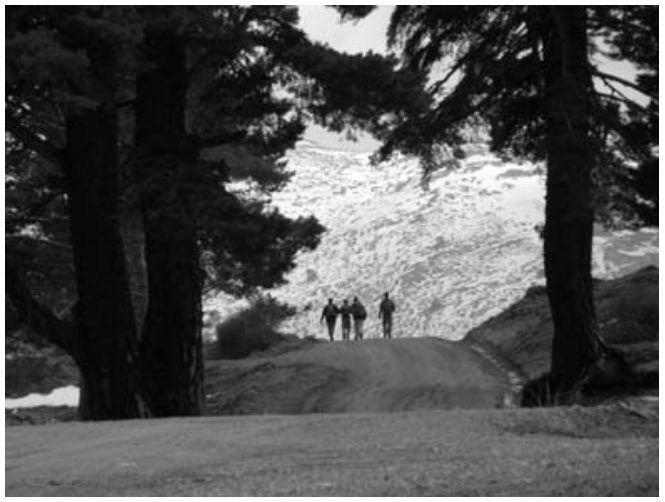

Figura 3 - Entorno en el que se desarrolló la parte práctica presencial de la actividad formativa. Parque Natural de la Sierra de Baza.

cada grupo una brújula, un mapa y una tarjeta de control. Actividad diseñada para realizarla en altitud y con grandes desniveles. A cada baliza señalada en el mapa se le asignó una puntuación determinada en función de la distancia al punto de salida, así como teniendo en cuenta la dificultad para hallarlo. La salida fue simultánea y se dio un tiempo máximo de dos horas para volver al punto de encuentro, cada grupo con los puntos conseguidos [figura 4].

Para la práctica de bicicleta [figura 5] se preparó un recorrido circular de unos treinta kilómetros por pistas y senderos, con distintas dificultades de conducción tanto técnica como físicamente, con tres planteamientos distintos de orientación a lo largo del recorrido. Los participantes tomaron la salida cronometrada con un periodo de tiempo entre grupos de tres minutos. En este caso, se contó con un vehículo de apoyo. A cada participante se entregó un mapa del itinerario, explicándoles en qué consistía la práctica, que se componía de tres formas distintas de trabajar la orientación. El primer tramo con la situación de las balizas señaladas en el mapa, a continuación un recorrido ciego, es decir, habiéndose borrado parte del mapa, con lo cual el participante no puede saber con lo que se va a encontrar y ha de tomar decisiones sobre el terreno para llegar a conectar con el siguiente punto del mapa que aparece tras el espacio blanco. En este tramo, las balizas han de hallarlas siguiendo las instrucciones o descripción del lugar que aparece en la hoja

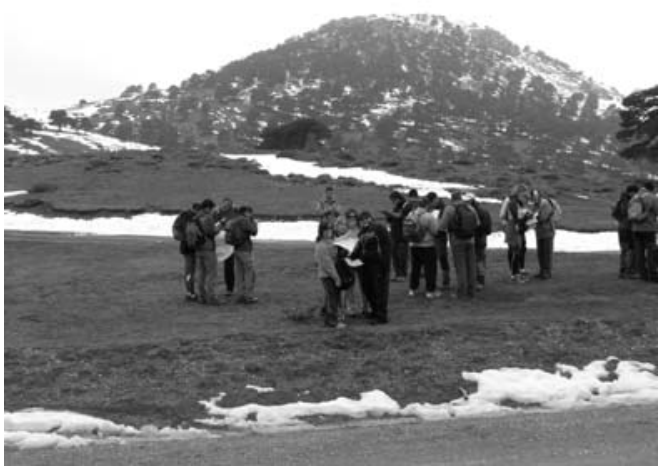

Figura 4 - En grupos de cuatro, preparándose para realizar "score orienting".

de control. Y por último, un tercer tramo en el que aparecen localizados los puntos donde se encuentran las balizas, pero no está trazado el recorrido sobre el plano. Para esta práctica, aparte de la indumentaria adecuada, también debían resolver cualquier incidencia mecánica que surgiera, por lo que además habían de ir perfectamente equipados para ello.

Para los contenidos del tercer bloque se preparó un cross de orientación con nueve estaciones distintas, señaladas en el mapa, con la salvedad de que en cada una de ella tenían que resolver distintas situaciones relacionadas con los diferentes contenidos trabajados en este bloque. A cada grupo se asignó una estación por la que debía iniciar su recorrido. En cada estación se situó un colaborador con instrucciones precisas de la actividad que debían realizar los participantes una vez llegaran los cuatro componentes del grupo. Así, una vez finalizada dicha actividad, el colaborador firmaría la hoja de control, dando fe de que había superado la prueba. Éstas eran de distinto carácter y complejidad, así por ejemplo había que realizar: tiro con arco (en la que en función de los puntos conseguidos por cada grupo se restarían determinados segundos al tiempo total empleado), circuito de habilidad en bicicleta por la montaña, montaje y desmontaje de tiendas de campaña, técnica descenso y descenso con mochilas, montar y desmontar un vivac, cabuyería, etc. A cada grupo se entregaba al inicio un mapa con las estaciones

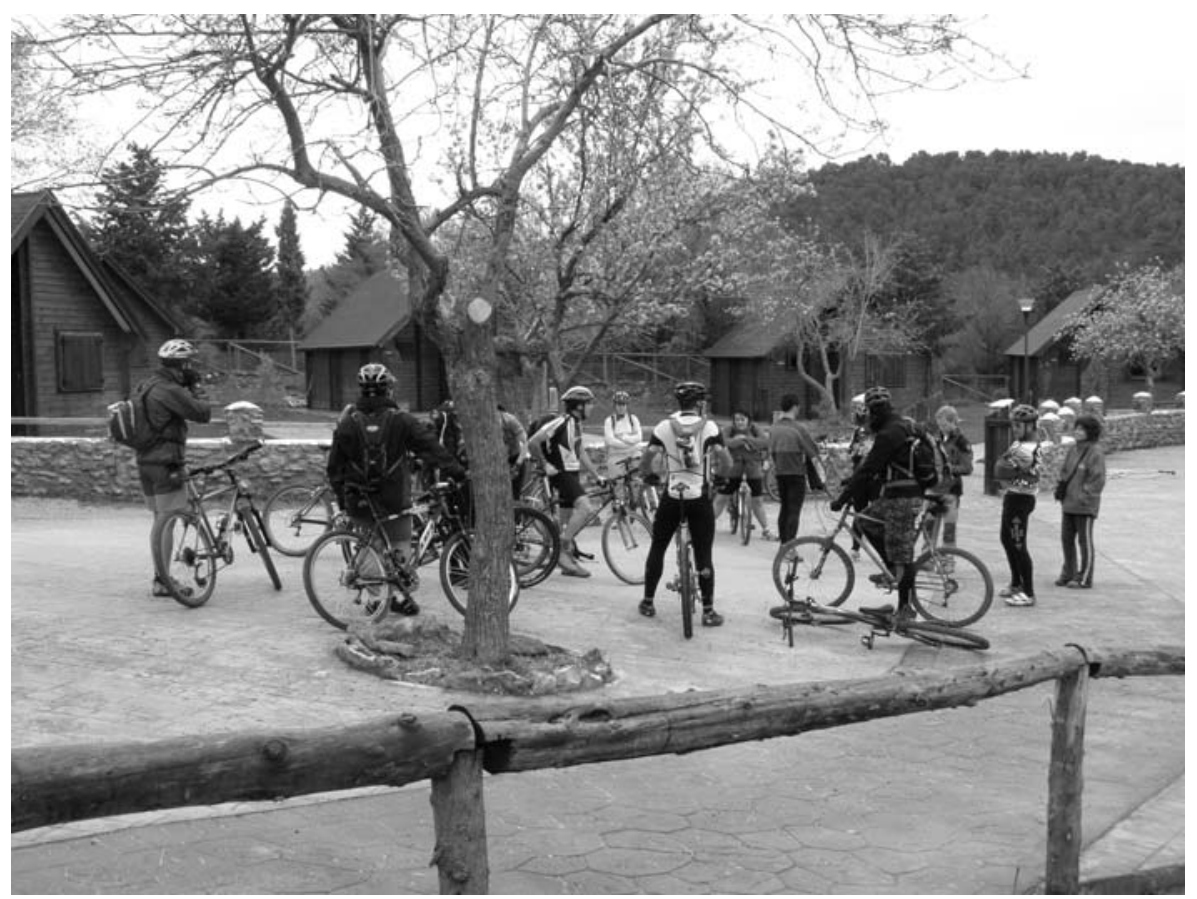

Figura 5 -Preparándose para realizar la práctica en bicicleta. 
Tabla 1 - Valoración porcentual de distintas variables relacionadas con los contenidos.

\begin{tabular}{ccccccc}
\hline \multirow{2}{*}{ V 1 } & & Muy poca & Poca & Adecuada & Mucha & Excesiva \\
\cline { 3 - 7 } & Cantidad de los contenidos & 0 & 0 & 54,29 & 28,57 & 17,14 \\
& Dificultad de los contenidos & 0 & 2,86 & 71,43 & 22,86 & 2,86 \\
\cline { 3 - 7 } & & Muy poca & Poca & Normal & Buena & Excelente \\
\cline { 3 - 7 } V2 & Calidad de los contenidos & 0 & 0 & 2,86 & 34,29 & 62,86 \\
& Utilidad de los contenidos & 0 & 0 & 2,86 & 51,43 & 45,71 \\
\hline
\end{tabular}

Tabla 2 - Valoración porcentual de los materiales del curso en relación a las expectativas iniciales.

\begin{tabular}{ccccccc}
\hline & NS/NC & $\begin{array}{c}\text { Decep- } \\
\text { cionantes }\end{array}$ & $\begin{array}{c}\text { Poco } \\
\text { adecuados }\end{array}$ & Adecuados & $\begin{array}{c}\text { Mejor de lo } \\
\text { esperado }\end{array}$ & $\begin{array}{c}\text { Extra- } \\
\text { ordinarios }\end{array}$ \\
\cline { 2 - 7 } $\begin{array}{c}\text { Materiales relación a } \\
\text { expectativas iniciales }\end{array}$ & 2,86 & 2,86 & 0 & 14,29 & 45,71 & 34,29 \\
\hline
\end{tabular}

señaladas, sin recorrido determinado, sólo se les indicaba la estación por la que habían de iniciar el recorrido, pero a continuación, ellos elegían su propio itinerario, hasta completar todo el cross.

\section{Evaluación de la actividad formativa por los participantes}

El curso lo iniciaron 90 especialistas de Educación Física de primaria y secundaria, superándolo un total de 81 personas. Al finalizar la actividad formativa, tras la realización de la práctica, el profesorado participante completó una encuesta anónima a través de la plataforma utilizada para la formación on-line para evaluar el curso en su totalidad. Fueron distintas las variables valoradas, aunque se echa en falta una selección más adecuada de las cuestiones, pues se plantearon las mismas para todos los cursos realizados a través de la plataforma CEPIndalo, independientemente de la temática, y es probable que las preguntas realizadas al profesorado de Educación Física que realizó este curso hubieron de ser más precisas y adecuadas a la actividad. En cualquier caso, las respuestas de evaluación dadas por los participantes arrojan datos de gran interés, dadas las características de la misma.

La valoración que se efectúa respecto a los contenidos tratados muestran que tanto la cantidad como la dificultad de los mismos ha resultado adecuada para la mayoría del profesorado participante, aunque una parte importante manifiesta que ha sido mucha o excesiva. Es probable que gran parte de este porcentaje corresponda a maestros y maestras de primaria, cuya formación inicial es menos profunda que la del profesorado de secundaria especialista en Educación Física. Asimismo, es realmente destacable que, a pesar de que los contenidos teóricos del curso se hayan impartido y trabajado on-line, las dos terceras partes consideren la calidad de los contenidos de excelente, así como los porcentajes (prácticamente la totalidad) de los que expresan la utilidad y aplicabilidad de los contenidos [tabla 1].
También resulta interesante conocer el cumplimiento de las expectativas, en relación con los materiales trabajados en el curso, una vez finalizada la actividad. Destacan los porcentajes que reflejan que estos materiales han resultado «mejor de lo esperado» $(45,71 \%)$ o «extraordinarios» $(34,29 \%)$ [tabla 2].

Otro aspecto a destacar positivamente de esta experiencia formativa es la implicación y compromiso del profesorado participante en la misma, tanto en la parte teórica como, por supuesto, en la práctica, como se puede observar en la figura 6, mostrando, en todo momento, una actitud participativa y dinamizadora $(92 \%)$.

Resulta de gran importancia recabar datos referentes a este tipo de formación en comparación con la acción formativa presencial, y más aún si se tiene en cuenta que se tratan de contenidos de actividades físicas en el medio natural, dirigidos a la formación de profesorado especialista en Educación Física. Como se puede observar en la tabla 3, más de ocho de cada diez participantes como alumnos y alumnas en este curso considera que la teleformación favorece el aprendizaje, destacando entre los mismos un elevado porcentaje $(22,86 \%)$ que estiman que favorece extraordinariamente el aprendizaje, en comparación con la clásica y tradicional formación presencial. De igual manera, prácticamente todos manifiestan seguridad en recomendar este tipo de formación a un compañero o compañera.

También es importante conocer la valoración global de la actividad formativa y realizada en referencia a distintas variables. Como se comprueba en los datos de la tabla 4, es de destacar la valoración que hace referencia a los contenidos trabajados a lo largo del curso, ya que más de dos terceras partes del alumnado los considera muy buenos, siendo necesario sumarle, además, un $20 \%$ que los estima como buenos. La valoración positiva de estos datos guardan relación con la tutorización, ya que el trabajo de los tutores, evaluado por más del $90 \%$ como bueno o muy bueno, obtiene su recompensa también en la evaluación de los

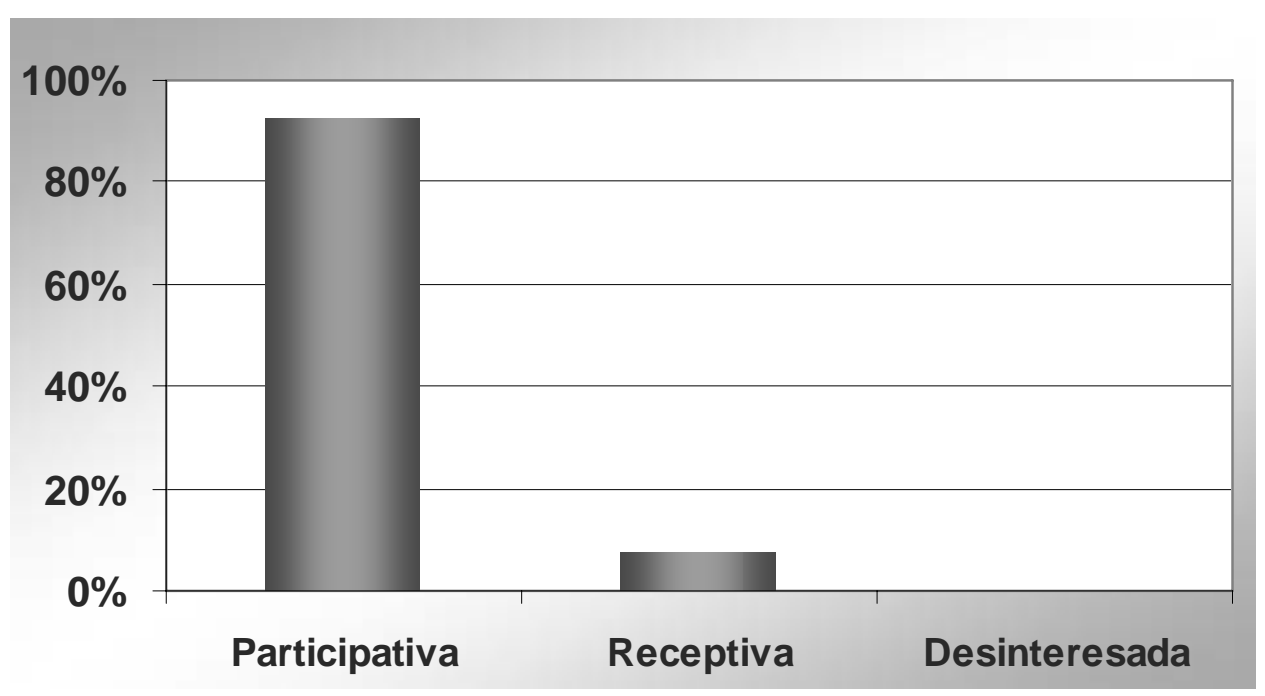

Figura 6 - Valoración de la participación e implicación del alumnado en el curso, por parte de los propios asistentes. 
Tabla 3 - Valoración, en función del aprovechamiento del curso, comparando el sistema de teleformación (on-line) con la acción formativa clásica presencial. Porcentajes.

\begin{tabular}{cccccc}
\hline & $\begin{array}{c}\text { Impide el } \\
\text { aprendizaje }\end{array}$ & $\begin{array}{c}\text { Dificulta el } \\
\text { aprendizaje }\end{array}$ & $\begin{array}{c}\text { No repercute } \\
\text { especialmente en el } \\
\text { aprendizaje }\end{array}$ & $\begin{array}{c}\text { Favorece } \\
\text { aprendizaje }\end{array}$ & $\begin{array}{c}\text { Favorece } \\
\text { extraordinariamente } \\
\text { el aprendizaje }\end{array}$ \\
\cline { 2 - 6 } $\begin{array}{c}\text { Teleformación vs acción } \\
\text { formativa presencial }\end{array}$ & 0 & 0 & 17,14 & 54,29 & 22,86 \\
\hline
\end{tabular}

Tabla 4 - Valoración global de distintas variables. Porcentajes.

\begin{tabular}{lccccc}
\hline \multicolumn{1}{c}{ Aspectos a valorar } & Muy malo & Malo & Aceptable & Bueno & Muy bueno \\
\hline Contenidos & 0 & 2,86 & 8,57 & 20 & 68,57 \\
Tutorización & 0 & 0 & 8,57 & 42,86 & 58,57 \\
Documentación & 0 & 0 & 11,43 & 20 & 68,57 \\
Actividades de la parte práctica presencial & 0 & 0 & 0 & 0 & 100 \\
Entorno de la parte práctica presencial & 0 & 0 & 0 & 0 & 100 \\
Coherencia entre la parte on-line y la práctica & 0 & 0 & 8,57 & 11,43 & 80 \\
Valoración global del curso & 0 & 0 & 8,57 & 42,86 & 48,57 \\
\hline
\end{tabular}

contenidos, así como de la documentación entregada y trabajada, cuya valoración prácticamente coincide con la de éstos. Un dato de gran relevancia y que llama la atención es que la totalidad (100\%) de los participantes en la actividad formativa valoran muy bien tanto las actividades de la parte práctica y presencial del curso, como el entorno en que fueron realizadas, obteniendo así la mayor consideración del profesorado participante, como alumnos en este caso. En línea con estos datos son los que se observan respecto a la coherencia entre la parte online y la práctica presencial, pues de cada diez, ocho (80\%) la consideran como muy buena, uno $(11,43 \%)$ como buena y otro $8,57 \%$ como aceptable. Así, por todos los participantes el curso es valorado positivamente desde un punto de vista global, destacando que la mitad (45,87\%) lo evalúan como muy bueno y el $42,86 \%$ como bueno. Por contra, prácticamente ninguna de las variables consideradas a evaluación han obtenido resultados negativos [figura 7].

Finalmente, considero interesante conocer algunas de las cuestiones que abiertamente han expresado los participantes en relación a los aspectos que más y menos le han gustado de esta actividad formativa, pues ponen de manifiesto las inquietudes y disposición del profesorado al afrontar y realizar este curso. Asimismo, resaltar que aunque se pueden echar en falta algunos aspectos negativos, no se ha dejado de reflejar nada en el apartado b), de las opiniones expresadas. A continuación se expone un resumen de los distintos testimonios:

a) Aspectos que más han gustado de este curso.

- «Las aportaciones de los participantes, en relación a las experiencias prácticas que han aportado a través de la plataforma».

- «Las aportaciones realizadas al foro». Referidas a la participación continua en el foro del curso por los distintos participantes.

- «Comodidad de horarios, flexibilidad, disponibilidad de material».

- «Estar en comunicación con los compañeros».

- «La ilusión de todos por mejorar nuestra docencia».

- «Los contenidos están tratados desde diferentes puntos de vista. La utilización de medios y canales hasta ahora poco habituales (red). Contenidos aplicados de forma concreta y experiencias muy diversasy contrastadas».

- «La facilidad para que cada uno vaya trabajando a su ritmo, la posibilidad de enriquecerse con las aportaciones de todos los compañeros».
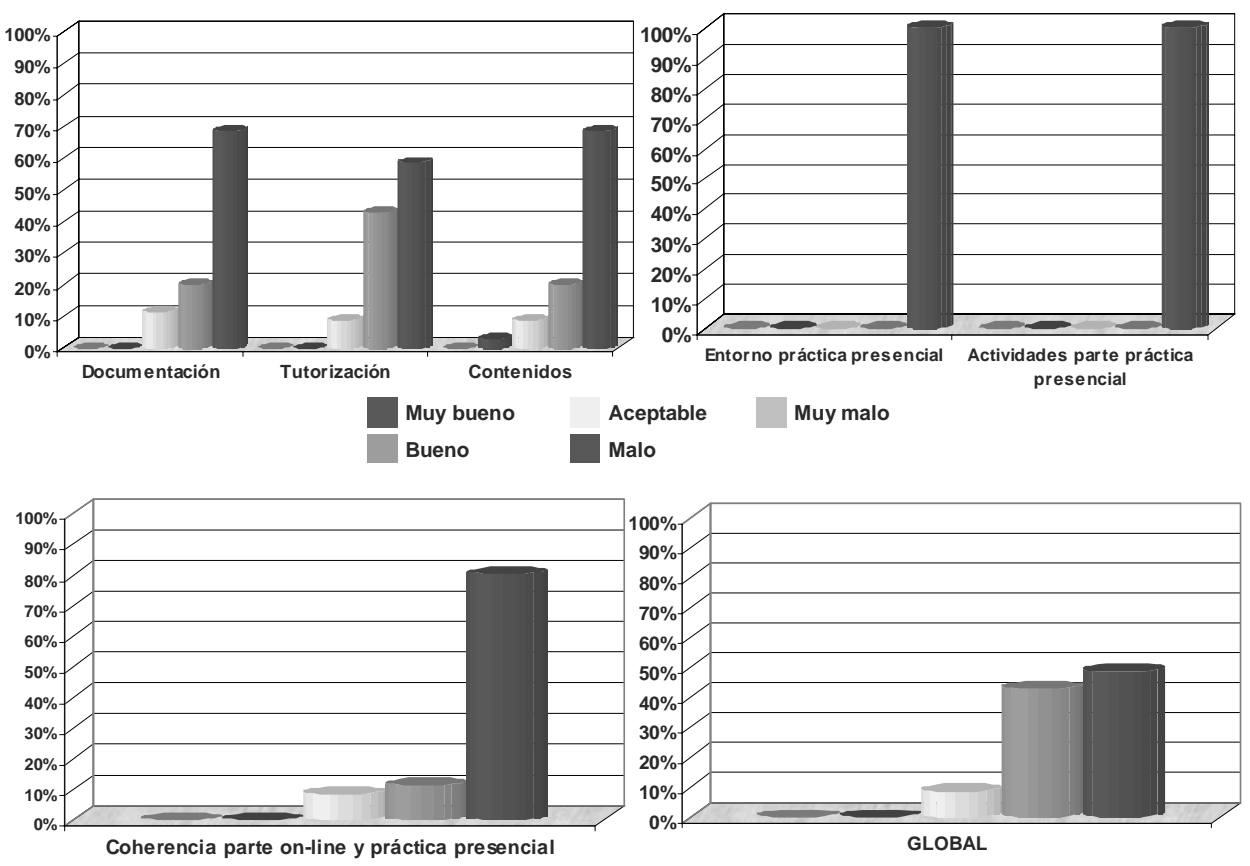

Figura 7 - Valoración de distintas variables y global del curso. 
b) Lo que menos ha gustado y las sugerencias de mejora, prácticamente coinciden y se pueden sintetizar en:

- «Accesibilidad a la plataforma educativa y descarga de los archivos». Esto se explica porque realmente a veces podía llegar a resultar frustrante si no se disponía de un ordenador potente y a la vanguardia en cuanto a características técnicas.

- «Simplemente pulir cuestiones referidas a la plataforma, poner la información un poco más clarita. Por lo demás, PERFECTO. Este curso cubrió SOBRADAMENTE mis expectativas, algo que hasta ahora no me había pasado en ningún otro curso del CEP».

\section{A modo de conclusión}

El profesorado de Educación Física, tanto de primaria como de secundaria, se siente motivado por la formación on-line, y participa activamente, al menos cuando el modelo está basado en el trabajo de contenidos de la especialidad, mostrando una actitud favorable a la innovación y un gran interés en formarse y contar con los recursos que ofrecen las nuevas tecnologías de la información y la comunicación para la preparación del alumnado. Asimismo, valora las virtudes de este tipo de formación en comparación con la presencial, tradicional y clásica, sobre todo por la libertad horaria que ofrece, individualización, intercambio de experiencias didácticas, etc. Incluso, el grado de satisfacción y aprovechamiento en esta actividad hace que la gran mayoría se muestre dispuesta a recomendar la teleformación entre los compañeros y compañeras de profesión.

A pesar de todo, el profesorado de esta materia, por las características de la misma, también necesita y quiere compartir experiencias prácticas con el resto de compañeros, valorando las relaciones personales y el trabajo desarrollado en grupo. Por ello, hay que tener en cuenta para futuros planteamientos el buen funcionamiento de este modelo de formación teórico-práctica on-line y con actividades prácticas (parte presencial) al final, realizadas con los demás participantes y donde se desarrollen distintos contenidos y planteamientos trabajados previamente a través de la red. Este encuentro final, además, servirá de convivencia y de intercambio de experiencias entre los participantes.

Aunque el tipo de contenidos influirá en el diseño de las actividades formativas, la experiencia aquí expuesta, teniendo en cuenta que se han trabajado distintos aspectos de las actividades en la naturaleza, que a priori no parece muy adecuado al modelo de teleformación, es un ejemplo de que, con una adecuada planificación y diseño, cualquier tipo de contenidos se puede adaptar a la formación on-line.

También es necesario apuntary destacar la importancia de la figura de los tutores o profesorado que imparte la actividad. Sobre ellos recae gran parte del resultado final, en este caso muy bueno, pues aunque el diseño de la actividad se realice en el Centro del Profesorado, ellos son los encargados de la elaboración y presentación de los contenidos, así como de la dinamización del trabajo del alumnado participante. La buena valoración del trabajo de los tutores es un indicador de que el curso ha resultado positivo y exitoso, cumpliendo las expectativas previas de los participantes.

\section{Referencias bibliográficas}

Cabero, J. Duarte, A. \& Barroso, J. (1997). La piedra angular para la incorporación de los medios audiovisuales, informáticos y nuevas tecnologías en los contextos educativos: la formación y el perfeccionamiento del profesorado. Revista Electrónica de Tecnología Educativa, $\mathrm{n}^{\circ}$ 8. Disponible en: http://www.uib.es/depart/gte/edutec/ -e/revelec8/revelec8.html. Consulta [2007, 12 de marzo].

Cebrián de la Serna, M.(1997). Nuevas competencias para la formación inicial y permanente del profesorado. Revista Electrónica de Tecnología Educativa, $\mathrm{n}^{\mathrm{o}}$ 6. Disponible en: http://www.uib.es/depart/ gte/edutec/-e/revelec8/revelec8.html. Consulta [2006, 22 de abril].

CEPIndalo (2007). Actividades de Teleformación de los CEP de la provincia de Almería. [en línea]. Disponible en http// aula.cepindalo.es/moodle/Consulta [2007, 25 de junio].

Decreto 110/2003 de 22 de abril, por el que se regula el SistemaAndaluz de Formación Permanente del Profesorado. Boletín Oficial de la Junta de Andalucía, 25 de abril de 2003, nº 78, p. 8591 - 8600. Sevilla.

Decreto 72/2003, de 18 de marzo, de Medidas de Impulso de la Sociedad del Conocimiento. Boletín Oficial de la Junta de Andalucía, 21 de marzo de 2003, nº 55, p. 6024 - 6033. Sevilla.

Foster, J. D., Campbell, W. K. \& Twenge, J. M. (2003). Individual differences in narcissism: Inflated self-views across the lifespan and around the world. Journal of Research in Personality, 37, 469486.

Majó i Cruzate, J. \& Marqués, P. (2002). La revolución educativa en la era de Internet. Barcelona: CISS-Praxis.

Marcelo, C. \& Estebaranz, A. (1999). Cultura escolar y cultura profesional: los dilemas del cambio. Revista Educar, 24, 47-147.

Orden de 9 de junio de 2003, por la que se aprueba el II Plan Andaluz de Formación Permanente. Boletín Oficial de la Junta de Andalucía, 26 de junio de 2003, nº 121, p. 14170 - 14178. Sevilla.

Palomo López, R., Ruiz Palmero, J. \& Sánchez Rodríguez, J. (2005). Las TIC como agentes de innovación educativa. Sevilla: Consejería de Educación de la Junta de Andalucía.

Real Decreto 1513/2006, de 7 de diciembre, por el que se establecen las Enseñanzas Mínimas correspondientes a la Educación Primaria. Boletín Oficial del Estado, $\mathrm{n}^{\circ} 293$ de 8 de diciembre de 2006.

Real Decreto 1631/2006, de 29 de diciembre, por el que se establecen las Enseñanzas Mínimas correspondientes a la Educación Secundaria Obligatoria. Boletín Oficial del Estado, $\mathrm{n}^{\mathrm{0}} 5$ de 5 de enero de 2007.

Twenge, J. M., Konrath, S., Foster, J. D., Campbell, W. K. \& Bushman, B. J. (2007). Egos inflating over time: A test of two generational theories of narcissism. Journal of Personality, (in press). Obtenido el 12 de julio de 2007, desde http://www.ksg.harvard.edu/saguaro/ pdfs/Twenge Narcissism 0207.pdf.

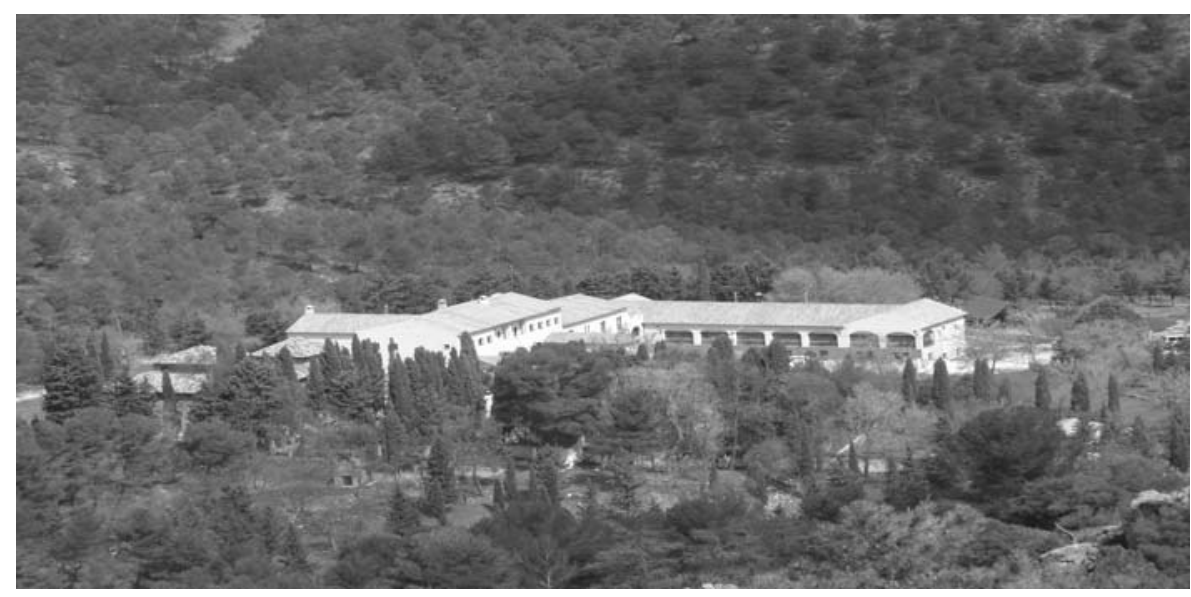

Parque Naturaldel la Sierra de Baza. Lugar donde se realizó la parte práctica presencial del curso. 